\title{
Kinetics of rare events for non-Markovian stationary processes and application to polymer dynamics
}

\author{
N. Levernier, ${ }^{1,2}$ O. Bénichou, ${ }^{3}$ R. Voituriez, ${ }^{3,4}$ and T. Guérin ${ }^{5}$ \\ ${ }^{1}$ Department of Theoretical Physics, University of Geneva, 1211 Geneva 4, Switzerland \\ ${ }^{2}$ Department of Biochemistry, University of Geneva, 1211 Geneva 4, Switzerland \\ ${ }^{3}$ Laboratoire de Physique Théorique de la Matière Condensée, CNRS/Sorbonne University, 4 Place Jussieu, F-75005 Paris, France \\ ${ }^{4}$ Laboratoire Jean Perrin, CNRS/Sorbonne University, 4 Place Jussieu, F-75005 Paris, France \\ ${ }^{5}$ Laboratoire Ondes et Matière d'Aquitaine, CNRS/University of Bordeaux, F-33400 Talence, France
}

(Received 26 July 2019; accepted 23 January 2020; published 10 March 2020)

\begin{abstract}
How much time does it take for a fluctuating system, such as a polymer chain, to reach a target configuration that is rarely visited-typically because of a high energy cost? This question generally amounts to the determination of the first-passage time statistics to a target zone in phase space with lower occupation probability. Here, we present an analytical method to determine the mean first-passage time of a generic non-Markovian random walker to a rarely visited threshold, which goes beyond existing weak-noise theories. We apply our method to polymer systems, to determine (i) the first time for a flexible polymer to reach a large extension, and (ii) the first closure time of a stiff inextensible wormlike chain. Our results are in excellent agreement with numerical simulations and provide explicit asymptotic laws for the mean first-passage times to rarely visited configurations.
\end{abstract}

DOI: 10.1103/PhysRevResearch.2.012057

The first-passage time (FPT) quantifies the time required for a random walker to reach a "target" point [1-10], with applications in contexts as varied as finance, biophysics, search processes, or reaction kinetics [2]. In the case of systems with many internal degrees of freedom, such as polymers or membranes, the dynamics of a single degree of freedom, e.g., a reaction coordinate, is typically non-Markovian (i.e., displays memory effects), which significantly complexifies the theoretical description of their first-passage properties [10,11].

Generally speaking, one can distinguish between two classes of first-passage problems. First, the search for the target by the random walker can be limited by an "entropic" cost, such as in the case of a target located in a large confined domain, which has been the subject of many recent studies, both for Markovian [6-9,12,13], and non-Markovian [10,14,15] random walks. Second, a very different class of problems is the search for rarely visited configurations, i.e., limited by a high energy cost (or quasipotential cost in nonequilibrium systems [16-18]). Such a problem is the cornerstone of reaction rate theory $[19,20]$, but is also crucial in situations as varied as population [21] or disease [22] extinction, bond rupture [23-25], adhesion [26], stock market crashes [27], or extreme heat waves in climate models [28]. The kinetics of rare events has been intensively investigated, and explicit expressions have been proposed for the noise-induced escape time from attraction domains in the weak-noise limit [19,29-34]. However, for non-Markovian processes, existing approaches fail to

Published by the American Physical Society under the terms of the Creative Commons Attribution 4.0 International license. Further distribution of this work must maintain attribution to the author(s) and the published article's title, journal citation, and DOI. predict quantitatively the first-passage time to a generic rarely visited target, such as a threshold for a reaction coordinate. For example, in the context of large deviation kinetics of flexible polymers, it has recently [35] been noted that standard weak-noise theories (to be defined below) lead to erroneous scalings for the mean FPT.

In this Rapid Communication, we investigate the impact of memory effects on the mean time a continuous nonMarkovian (possibly non-Gaussian) variable $r(t)$ takes to reach a given rarely visited threshold. We show that memory effects can be accounted for by characterizing the trajectory followed by $r(t)$ in the future of the FPT, which generalizes a recent theoretical approach restricted to unbiased Gaussian processes [15]. We obtain explicit asymptotic expressions of the mean FPT to a rarely visited target, in excellent agreement with simulations. Our analysis reveals that memory effects, which so far have been left aside for this situation, can modify the kinetics by more than one order of magnitude, and finally provides a refined characterization of the dynamics of visits to rare configurations for generic stationary non-Markovian processes.

We illustrate our methodology by solving two problems involving polymer chains (Fig. 1), which provide prototypical examples of physical systems with many interacting degrees of freedom, where reaction coordinates thus display memory effects [36-38]. We calculate the mean time for a flexible chain to spontaneously reach a large extension, which is relevant in ligand adhesion via flexible tethers [26] and for the rheology of entangled melts $[35,39,40]$. We also investigate the closure kinetics of a stiff wormlike chain (i.e., a fluctuating thin rod). While this problem is highly relevant in the context of DNA looping kinetics [41], it has so far seemed analytically intractable, notably due to the difficulty to describe the non-Gaussian stochastic dynamics of highly 
(a)

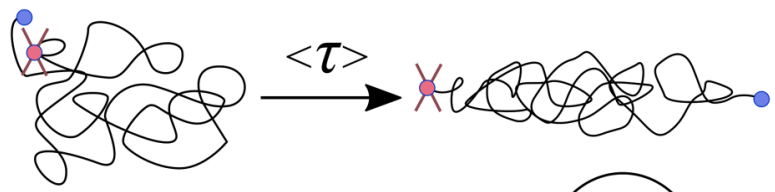

(b)

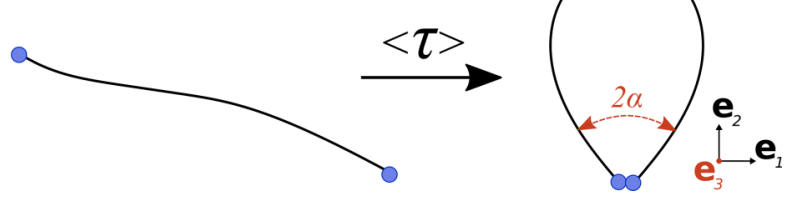

FIG. 1. What is the mean first time to reach a rare configuration? This Rapid Communication investigates this question in the case of (a) an attached flexible polymer, for which we compute the time that a large extension is reached, and (b) a stiff wormlike chain, for which we compute the time that the extremities get into contact.

curved rods. Existing theories for this problem rely either on mean-field approximations $[42,43]$ or on a mapping to one-dimensional (1D) dynamics [44-47], which disagree with numerical simulations [48].

First passage for an attached flexible polymer. We first consider the simplest model of flexible polymer, formed by $N$ phantom beads, with friction coefficient $\gamma$, linked by springs of stiffness $k$. The overdamped evolution of the beads' positions $x_{i}(i$ is the bead index) follows from a force balance [38]

$$
\gamma \dot{x}_{i}=k\left(x_{i+1}-2 x_{i}+x_{i-1}\right)+f_{i}(t),
$$

where thermal forces obey $\left\langle f_{i}(t) f_{j}\left(t^{\prime}\right)\right\rangle=2 k_{B} T \gamma \delta\left(t-t^{\prime}\right) \delta_{i j}$. We denote by $l_{0}=\sqrt{k_{B} T / k}$ the typical bond length, and $\tau_{0}=\gamma / k$ the typical relaxation time of a single bond. The first monomer is fixed, $x_{1}=0$, and we study the mean time $\langle\tau\rangle$ that the other polymer end $r(t)=x_{N}(t)$ reaches a threshold value $z$ [Fig. 1(a)]. The energy at fixed $z$ is given by $U=k z^{2} /(2 N)$, and we assume $U \gg k_{B} T$, so that first-passage events to $z$ are rare.

Figure 2(a) shows the mean FPT obtained from simulations results of Ref. [35] and existing analytical approximations for a fixed and relatively high value of the energy $\operatorname{cost} U \simeq$ $18 k_{B} T$. Substantial disagreement that increases with $N$ is found, be it for adiabatic approximations [35,52], effective one-dimensional descriptions $[39,40]$, and even the rigorous weak-noise approach [ $T \rightarrow 0$ at fixed $N$-see Refs. [30,35] and the Supplemental Material (SM) [49]]. This shows the necessity to take into account the collective dynamics of all monomers to calculate the mean FPT, which is the main purpose of this Rapid Communication. In fact, the nonMarkovian theory that we introduce here shows an excellent agreement with simulations [Fig. 2(a)], which holds for a broad range of values of the energy barrier [Fig. 2(b)].

General expressions for the mean FPT. We now consider the more general problem of the FPT of a stochastic (onedimensional) variable $r(t)$ to a rarely visited threshold $z$. We assume that $r(t)$ is nonsmooth [10], meaning that $\left\langle\dot{r}^{2}\right\rangle=\infty$, as is the case for overdamped processes. We denote $p(r, t)$ the probability density distribution of $r$ at time $t$, starting from a given initial position $r_{0}$ that will be proved to be irrelevant. We also assume that $r(t)$ is stationary at long times, $p(r, t) \underset{t \rightarrow \infty}{\rightarrow}$ $p_{s}(r)$, where the stationary distribution $p_{s}(r)$ is reached after a
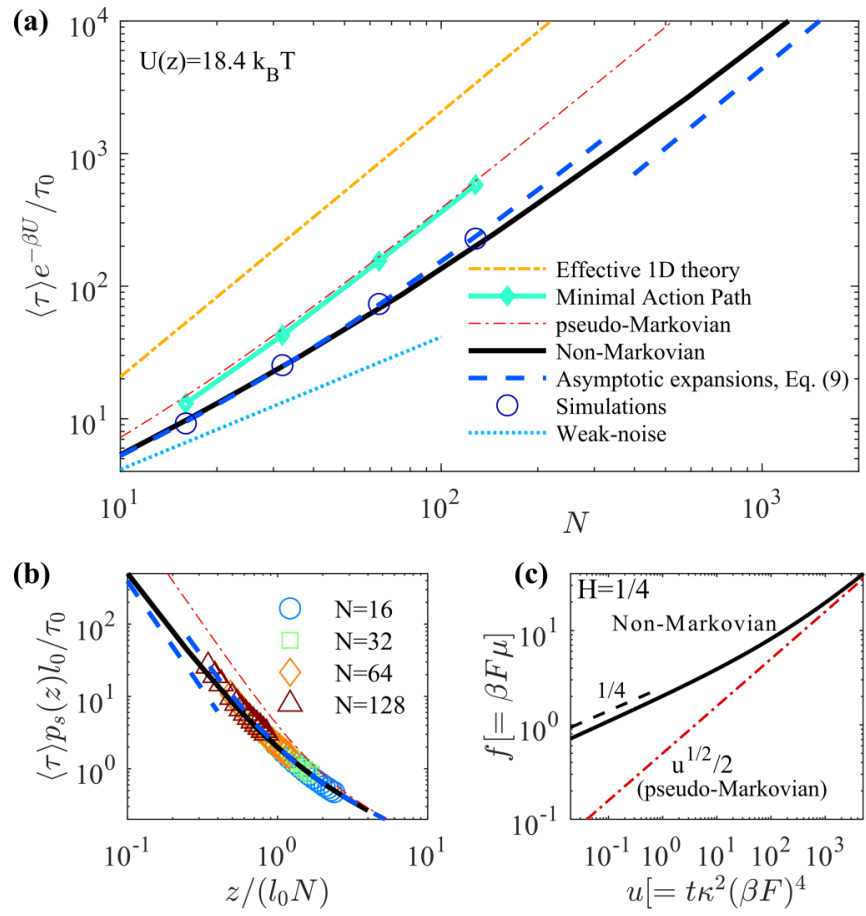

FIG. 2. (a) Mean FPT for a flexible chain to reach an extension $z=3.5 l_{0} \sqrt{3 N}$, corresponding to a fixed energy cost $U=$ $18.4 k_{B} T$. Symbols: Simulations of Ref. [35]. Different curves correspond to different theories, obtained (from top to bottom) via a mapping over 1D dynamics (Milner-McLeish reptation theory $[39,40]$, upper dashed line), the minimal action path method [35], the pseudo-Markovian (Wilemski-Fixman [52]) approximation, the non-Markovian theory (this work, black thick line), asymptotic expansions of the non-Markovian theory [dashed blue line, Eq. (9), this work], and the weak-noise result $T \rightarrow 0$, fixed $N$ [30,35]. Details on all theories can be found in SM [49]. (b) Mean FPT in rescaled variables, with supplemental simulation data of Ref. [35] (symbols). Lines share the same color code as in (a). (c) Rescaled average trajectory $\mu(t)$ in the future of the FPT for a scale invariant process with $H=1 / 4$. The dashed red line would be the future trajectory by assuming equilibrium at initial time.

finite correlation time $t_{c}$. With these hypotheses, the following exact expression can be obtained [15],

$$
\langle\tau\rangle p_{s}(z)=\int_{0}^{\infty} d t\left[p_{\pi}(z, t)-p(z, t)\right],
$$

where $p_{\pi}(r, t)$ is the probability density of $r$ at a time $t$ after the first passage. Now, in the case of targets that are only rarely visited, we stress the following key points: (i) As long as $r_{0}$ is not in the close vicinity of $z, p(z, t)$ is exponentially small (with noise intensity) at all times, and (ii) the probability $p_{\pi}(z, t)$ to revisit the target after a time $t$ is exponentially small at long times, but finite at times that immediately follow a FPT event, when $r$ is still close to $z$. The integral (2) is dominated by this short-time contribution, where $p_{\pi}$ can be replaced by its value $p_{\pi}^{\infty}(z, t)$ obtained by considering the linearized dynamics around the target point. Hence, the mean FPT to a rare configuration is asymptotically (rare event limit) given by

$$
\langle\tau\rangle p_{s}(z) \simeq \int_{0}^{\infty} d t p_{\pi}^{\infty}(z, t)
$$


Since $p_{\pi}^{\infty}(z, t)$ is a return probability for a particle submitted to a constant force in infinite space, it vanishes fast enough at long times so that expression (3) is defined without any ambiguity. Note that for rare events the initial distribution of $r$ has typically been forgotten long before the FPT, and thus does not influence $\langle\tau\rangle$. The above equation suggests a two-step strategy to obtain $\langle\tau\rangle$. The first step consists in characterizing the static quantity $p_{s}(z)$; for equilibrium systems one obtains $p_{s}(z) \propto e^{-U(z) / k_{B} T}$ and in particular $\langle\tau\rangle$ follows an Arrhenius-like law [53]. The second step consists in analyzing the dynamics of $r(t)$ in the vicinity of the target $z$ to deduce $p_{\pi}^{\infty}(z, t)$.

To proceed further, we assume that the dynamics of $r(t)$ near $z$ is Gaussian, which is valid in the vicinity of the most probable configuration. We denote by $m_{s}(t)$ and $\psi(t)$, respectively, the mean and the variance of $r(0)-r(t)$ when the initial state is the stationary distribution conditional to $r(0)=z$. We adapt the theory of Ref. [15] (restricted to unbiased dynamics), based on the hypothesis that the trajectories followed by the random walker in the future of the FPT display Gaussian statistics. Defining the average future trajectory as $\langle r(t+\mathrm{FPT})\rangle=z-\mu(t)$ and approximating the variance in the future of the FPT by $\psi(t)$, we can write the so-far unknown quantity $p_{\pi}^{\infty}(z, \tau)$ as

$$
p_{\pi}^{\infty}(z, t)=[2 \pi \psi(t)]^{-1 / 2} e^{-\mu(t)^{2} / 2 \psi(t)} .
$$

The average future trajectory $\mu(t)$ itself satisfies the selfconsistent integral equation (see SM [49])

$$
\begin{aligned}
& \int_{0}^{\infty} d t \frac{e^{-\mu(t)^{2} /[2 \psi(t)]}}{\psi(t)^{1 / 2}}\{\mu(t+\tau) \\
& \left.-\mu(t) \frac{\psi(t+\tau)+\psi(t)-\psi(\tau)}{2 \psi(t)}-m_{s}(\tau)\right\}=0 .
\end{aligned}
$$

We note that our theory holds for general nonequilibrium systems. Here, we focus on equilibrium ones, in which case the fluctuation-dissipation theorem imposes

$$
m_{s}(t)=-\frac{F \psi(t)}{2 k_{B} T},
$$

where $F=-\partial_{z} U(z)$. For the Markovian (diffusive) case with $\psi(t) \propto t$, there is an obvious solution $\mu(t)=m_{s}(t)$. For nonMarkovian variables, this relation does not hold and the future trajectory $\mu(t)$ reflects the state of the nonreactive degrees of freedom at the FPT. Finally, Eqs. (3)-(5) fully define the mean FPT to a rare configuration for general non-Markovian processes that are locally Gaussian.

In the case of a biased anomalous dynamics with $\psi(t)=$ $\kappa t^{2 H}$, where $0<H<1$, Eq. (5) predicts that $\mu$ takes the scaling form

$$
\mu(t)=-\frac{k_{B} T}{F} f\left(t\left(\frac{\sqrt{\kappa}|F|}{k_{B} T}\right)^{1 / H}\right),
$$

and the mean FPT reads

$$
\langle\tau\rangle p_{s}(z)=\frac{A_{H}\left(k_{B} T\right)^{\frac{1-H}{H}}}{|F|^{\frac{1-H}{H}} \kappa^{\frac{1}{2 H}}}, \quad A_{H}=\int_{0}^{\infty} d u \frac{e^{-\frac{f^{2}(u)}{2 u^{2 H}}}}{\sqrt{2 \pi} u^{H}} .
$$

This formula provides an explicit asymptotic relation for the mean FPT, as a function of the subdiffusion coefficient $\kappa$, the local force $F$, and the temperature $k_{B} T$, and $A_{H}$ depends only on $H$ ( $f$ is defined in SM [49]). Of note, this result (8) is consistent with the scaling proposed in Ref. [55] for processes that are Gaussian (not only locally). In addition, it agrees with the more recent derivation of the prefactor for this scaling based on a perturbative scheme [56] in $\varepsilon \equiv H-1 / 2$ (see SM [49]). We now discuss applications of these general results.

Application to the kinetics of large extension for a flexible chain. Let us come back to the above example of an attached flexible chain. It is well known that the dynamics of the ends is either diffusive, $\psi(t)=2 D_{0} t$ for $t \ll \tau_{0}$, or subdiffusive, $\psi(t)=\kappa t^{1 / 2}$ with $\kappa=4 k_{B} T /(\pi \gamma k)^{1 / 2}$ when $\tau_{0} \ll t \ll t_{c}$, where $t_{c}=N^{2} \tau_{0}$ is the correlation time. The mean FPT is controlled either by the short-time diffusive regime $(H=1 / 2)$ or by the intermediate subdiffusive regime $(H=1 / 4)$, so that

$$
\langle\tau\rangle p_{s}(z)=\frac{\tau_{0}}{l_{0}} \times\left\{\begin{array}{lc}
0.39\left(\frac{N l_{0}}{z}\right)^{3} & \left(l_{0} \sqrt{N} \ll z \ll l_{0} N\right), \\
\frac{N l_{0}}{z}\left[1+\left(\frac{N l_{0}}{z}\right)^{2}\right] & \left(N l_{0} \ll z\right),
\end{array}\right.
$$

where we have included the (asymptotically exact) next-toleading-order expansion in the large $z$ limit (which coincides with the weakly non-Markovian limit—see SM [49]). This expression incorporates non-Markovian effects that were neglected in Ref. [35]. Here, we have used the value $A_{1 / 4}=2.0$, which we obtained by numerically solving Eq. (5). This value is about eight times smaller than in the pseudo-Markovian approximation (where $\mu \simeq m_{s}$, leading to with $A_{1 / 4}^{\mathrm{WF}}=16$ ). Here, the memory effects are nearly of one order of magnitude for the mean FPT and are thus strong. This originates from the qualitative difference between the short-time behavior of the trajectory after the first passage $\mu(t) \sim t^{1 / 4}$ and that of $m_{s}(t) \sim t^{1 / 2}$ (following the stationary state with $z=r$ ) [Fig. 2(c)]. At short times $\mu(t)$ can therefore be infinitely larger than $m_{s}(t)$, which means that local equilibrium assumptions are inaccurate in this situation. All data of the mean FPT can be collapsed on a single master curve depending only on $z / l_{0} N$, with asymptotics given by Eq. (9). This is done in Fig. 2(b), where we see that the simulation data closely follow (but are slightly larger than) our theoretical predictions. Finally, our theory provides an accurate description of the kinetics with which a flexible polymer reaches a large extension.

The closure time of a stiff wormlike chain. We now consider a thin inextensible elastic rod with bending rigidity $\kappa_{b}$. In the stiff limit, where the persistence length $l_{p}=\kappa_{b} / k_{B} T$ is much larger than the contour length $L$, closure events are rare since they require overcoming a large bending energy barrier. Here, we calculate the closure time $\langle\tau\rangle$ defined as the mean time for the end-to-end distance $r$ to reach a value $a \ll L$. We assume the dynamics to be described by the resistive force theory, in which viscous forces apply locally on the filament with friction coefficients per unit length $\zeta_{\perp}, \zeta_{\|}$(respectively in the parallel and perpendicular directions) $[57,58]$. We furthermore assume that no force and no torque are exerted at the chain ends.

Determining the closure time [Eq. (3)] first requires one to calculate $p_{s}(r)$, which is an equilibrium (static) statistical mechanics problem which has been studied at length by a variety of analytical and numerical methods [59-63]. It is also needed to characterize the dynamics at the early times 


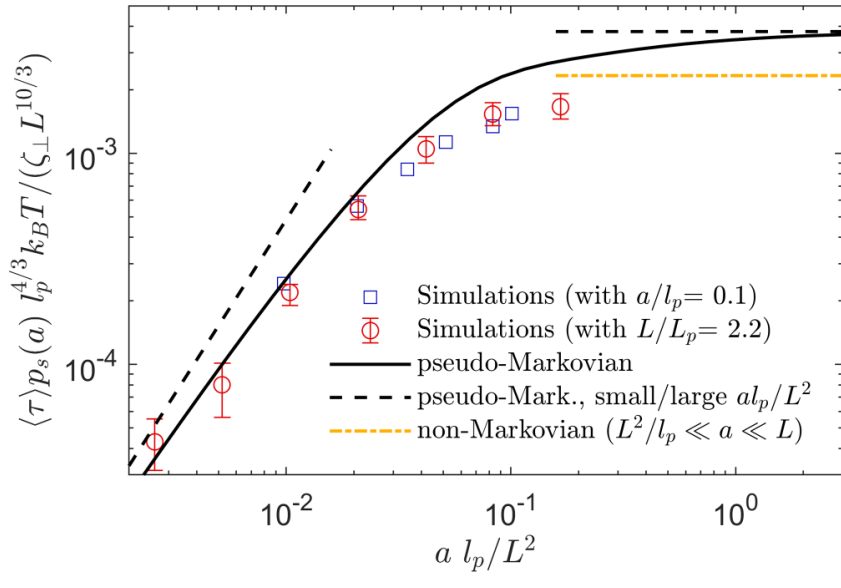

FIG. 3. Mean closure time for wormlike chains as a function of capture radius $a$, shown in rescaled variables. Symbols: Simulations of Ref. [48], rescaled by $p_{s}(r)$ given in Ref. [63]. Continuous black line: Pseudo-Markovian approximation [Eq. (11)], with asymptotic regimes indicated by the dashed black lines. Dashed-dotted orange line: Non-Markovian theory for $L^{2} / l_{p} \ll a \ll L$, Eq. (12).

following a closure event. Such dynamics necessarily occurs at the vicinity of the close configurations of minimal bending energy. Of note, lateral fluctuations are of the order of $\ell_{\perp}(t) \propto$ $t^{1 / 4}[58,64]$ which is small at short times. This key remark implies that the essential of the dynamics after closure takes place near the extremities, where the chain can be considered as close to a straight rod. We can then calculate analytically the evolution of the end-to-end vector when initial conditions are closed equilibrium configurations. Characterizing this dynamics in the reference frame $\left\{\mathbf{e}_{i}\right\}$ defined by the configuration at closure [see Fig. 1(b)] as $\mathbf{r}_{\mathrm{ee}}(t)=\mathbf{r}_{\mathrm{ee}}(0)+\sum_{i=1}^{3} X_{i}(t) \mathbf{e}_{i}$, we obtain (see SM [49])

$$
\begin{aligned}
\left\langle X_{1}(t)\right\rangle & =\frac{F \kappa t^{3 / 4} \cos ^{2} \alpha}{2 k_{B} T}, \quad \kappa=\frac{4 \sqrt{2} k_{B} T}{\Gamma(7 / 4) \zeta_{\perp}^{3 / 4} \kappa_{b}^{1 / 4}}, \\
\operatorname{Cov}\left[X_{i}(t), X_{j}(t)\right] & =\left(\begin{array}{ccc}
\cos ^{2} \alpha & 0 & 0 \\
0 & \sin ^{2} \alpha & 0 \\
0 & 0 & 1
\end{array}\right) \kappa t^{3 / 4},
\end{aligned}
$$

where $\alpha$ is half the opening angle of the most probable closed configurations (Fig. 1), and the force is $F=21.55 \kappa_{b} / L^{2}=$ $-U^{\prime}(0)$, with $U(a)$ the energy cost to form a closed configuration. The stationary dynamics around a closed configuration is thus a three-dimensional biased anisotropic subdiffusion. Note that $\left\langle X_{1}(t)\right\rangle$ and $\operatorname{Var}\left(X_{1}\right)$ are again linked by the ratio $F / 2 k_{B} T$, which is consistent with the fluctuation-dissipation theorem. A first estimate of the closure time can be obtained by assuming $p_{\pi}(t) \simeq p(a, t \mid a, 0)$ (pseudo-Markovian approximation). This can be readily calculated from the Gaussian dynamics specified by Eq. (10), leading to

$$
\langle\tau\rangle p_{s}(a)=\frac{\zeta_{\perp} L^{10 / 3}}{k_{B} T l_{p}^{4 / 3}} \Phi\left(\frac{a l_{p}}{L^{2}}\right),
$$

where $\Phi$ is a scaling function calculated in SM [49] represented in Fig. 3 (black line). This figure also displays the simulation data of Ref. [48], which collapse as in Eq. (11) onto a curve which is close to $\Phi$ for small arguments. We stress that there is no fitting parameter in the theory.

However, there is a difference of a factor of about 2 between theory and numerics for a larger capture radius, suggesting that non-Markovian effects are significant in the regime $a \gg L^{2} / l_{p}$, which we investigate now (while still keeping the small capture radius condition $a \ll L$ ). In this case the dynamics needs to be characterized only at timescales where the return probability is not exponentially small, i.e., such that $\left\langle X_{1}(t)\right\rangle^{2}$ is smaller than $\left\langle X_{1}^{2}(t)\right\rangle$. For these timescales, $X_{1} \sim L^{2} / l_{p}$ is still much smaller than $a$. This implies that the end-to-end distance is approximated at linear order as $r=\left[\left(a+X_{1}\right)^{2}+X_{2}^{2}+X_{3}^{2}\right]^{1 / 2} \simeq a+X_{1}$ and is thus equivalent to a one-dimensional Gaussian variable. The mean closure time can be obtained by applying the formalism presented above with $H=3 / 8$. We obtain

$$
\langle\tau\rangle p_{s}(a) \simeq 0.0023 \frac{\zeta_{\perp} L^{10 / 3}}{k_{B} T l_{p}^{4 / 3}} \quad\left(a \gg L^{2} / l_{p}\right) .
$$

Here, the value of the prefactor was obtained with $A_{3 / 8}=2.1$, which is 1.6 times smaller than its estimate in the pseudoMarkovian (Wilemski-Fixman) approximation $A_{3 / 8}^{\mathrm{WF}}=3.39$. This explains why the pseudo-Markovian theory overestimates the simulation data.

In the opposite limit $a \ll L^{2} / l_{p}$, the pseudo-Markovian expression (11) becomes

$$
\langle\tau\rangle p_{s}(a) \simeq 1.05 \frac{\zeta_{\perp} a^{5 / 3} l_{p}^{1 / 3}}{k_{B} T} \quad\left(a \ll L^{2} / l_{p}\right),
$$

and it can be shown that this result can be found by setting $F=0$, i.e., by analyzing a symmetric anisotropic threedimensional subdiffusive walk. In a recent work [43] for a similar (but isotropic) subdiffusive process, it was shown that memory effects led to a slight reduction $(15 \%)$ of the mean FPT. We expect a similar for the mean closure time, as confirmed by the comparison with numerical simulations in Fig. 3.

Conclusion. In this Rapid Communication, we have introduced theoretical tools to determine the mean FPT to rarely visited configurations for generic non-Markovian processes. We have derived explicit asymptotic expressions for the closure kinetics of a stiff wormlike chain, and for the mean FPT to a large extension of a flexible chain. As demonstrated by the example of wormlike chain closure, the dynamics needs to be Gaussian only locally (in the vicinity of the target) to apply our theory. This approach shows quantitatively the importance of memory effects on mean FPTs, and thereby significantly improves existing theories, whether based on a weak-noise limit, a mapping on one-dimensional problems or pseudo-Markovian (adiabatic) approximations. Our approach is not limited to polymers, and can apply to generic complex physical systems, where the dynamics of a reaction coordinate is coupled to many other degrees of freedom.

Acknowledgment. O.B. acknowledges the support of the European Research Council starting Grant No. FPTOpt277998. We thank A. Spakowitz for providing the data of Ref. [63]. 
[1] S. Redner, A Guide to First-Passage Processes (Cambridge University Press, Cambridge, UK, 2001).

[2] R. Metzler, S. Redner, and G. Oshanin, First-Passage Phenomena and their Applications (World Scientific, Singapore, 2014).

[3] A. Pal and S. Reuveni, Phys. Rev. Lett. 118, 030603 (2017).

[4] O. Bénichou, D. Grebenkov, P. Levitz, C. Loverdo, and R. Voituriez, Phys. Rev. Lett. 105, 150606 (2010).

[5] G. Vaccario, C. Antoine, and J. Talbot, Phys. Rev. Lett. 115, 240601 (2015).

[6] A. Godec and R. Metzler, Sci. Rep. 6, 20349 (2016).

[7] A. Godec and R. Metzler, Phys. Rev. X 6, 041037 (2016).

[8] S. Condamin, O. Bénichou, V. Tejedor, R. Voituriez, and J. Klafter, Nature (London) 450, 77 (2007).

[9] D. S. Grebenkov, Phys. Rev. Lett. 117, 260201 (2016).

[10] A. J. Bray, S. N. Majumdar, and G. Schehr, Adv. Phys. 62, 225 (2013).

[11] N. Van Kampen, Stochastic Processes in Physics and Chemistry, 3rd ed. (Elsevier, Amsterdam, 2007).

[12] O. Bénichou and R. Voituriez, Phys. Rev. Lett. 100, 168105 (2008).

[13] Z. Schuss, A. Singer, and D. Holcman, Proc. Natl. Acad. Sci. USA 104, 16098 (2007).

[14] T. Guérin, O. Bénichou, and R. Voituriez, Nat. Chem. 4, 568 (2012).

[15] T. Guérin, N. Levernier, O. Bénichou, and R. Voituriez, Nature (London) 534, 356 (2016).

[16] R. S. Maier and D. L. Stein, Phys. Rev. Lett. 69, 3691 (1992).

[17] M. I. Freidlin and A. D. Wentzell, Random Perturbations of Dynamical Systems (Springer, New York, 1984).

[18] R. de la Cruz, R. Perez-Carrasco, P. Guerrero, T. Alarcon, and K. M. Page, Phys. Rev. Lett. 120, 128102 (2018).

[19] P. Hänggi, P. Talkner, and M. Borkovec, Rev. Mod. Phys. 62, 251 (1990).

[20] E. Pollak and P. Talkner, Chaos 15, 026116 (2005).

[21] A. Kamenev, B. Meerson, and B. Shklovskii, Phys. Rev. Lett. 101, 268103 (2008)

[22] M. I. Dykman, I. B. Schwartz, and A. S. Landsman, Phys. Rev. Lett. 101, 078101 (2008).

[23] R. Merkel, P. Nassoy, A. Leung, K. Ritchie, and E. Evans, Nature (London) 397, 50 (1999).

[24] G. Hummer and A. Szabo, Biophys. J. 85, 5 (2003).

[25] J. T. Bullerjahn, S. Sturm, and K. Kroy, Nat. Commun. 5, 4463 (2014).

[26] C. Jeppesen, J. Y. Wong, T. L. Kuhl, J. N. Israelachvili, N. Mullah, S. Zalipsky, and C. M. Marques, Science 293, 465 (2001).

[27] J. P. Bouchaud and R. Cont, Eur. Phys. J. B 6, 543 (1998).

[28] F. Ragone, J. Wouters, and F. Bouchet, Proc. Natl. Acad. Sci. U.S.A. 115, 24 (2018).

[29] H. A. Kramers, Physica (Utrecht) 7, 284 (1940).

[30] Z. Schuss, Theory and Applications of Stochastic Processes: An Analytical Approach (Springer, Berlin, 2009), Vol. 170.
[31] F. Bouchet and J. Reygner, Ann. Henri Poincaré 17, 3499 (2016).

[32] R. F. Grote and J. T. Hynes, J. Chem. Phys. 73, 2715 (1980).

[33] P. Hänggi and F. Mojtabai, Phys. Rev. A 26, 1168(R) (1982).

[34] B. Meerson and A. Vilenkin, Phys. Rev. E 93, 020102(R) (2016).

[35] J. Cao, J. Zhu, Z. Wang, and A. E. Likhtman, J. Chem. Phys. 143, 204105 (2015).

[36] D. Panja, J. Stat. Mech.: Theor. Exp. (2010) P06011.

[37] J. T. Bullerjahn, S. Sturm, L. Wolff, and K. Kroy, Europhys. Lett. 96, 48005 (2011).

[38] M. Doi and S. F. Edwards, The Theory of Polymer Dynamics (Oxford University Press, New York, 1988).

[39] S. T. Milner and T. C B. McLeish, Phys. Rev. Lett. 81, 725 (1998).

[40] S.T. Milner and T .C B. McLeish, Macromolecules 30, 2159 (1997).

[41] R. Vafabakhsh and T. Ha, Science 337, 1097 (2012).

[42] A. Dua and B. Cherayil, J. Chem. Phys. 116, 399 (2002).

[43] T. Guérin, M. Dolgushev, O. Bénichou, R. Voituriez, and A. Blumen, Phys. Rev. E 90, 052601 (2014).

[44] S. Jun, J. Bechhoefer, and B.-Y. Ha, Europhys. Lett. 64, 420 (2003).

[45] C. Hyeon and D. Thirumalai, J. Chem. Phys. 124, 104905 (2006).

[46] J. Chen, H. Tsao, and Y. Sheng, Europhys. Lett. 65, 407 (2004).

[47] T. T. Le and H. D. Kim, Biophys. J. 104, 2068 (2013).

[48] R. Afra and B. A. Todd, J. Chem. Phys. 138, 174908 (2013).

[49] See Supplemental Material at http://link.aps.org/supplemental/ 10.1103/PhysRevResearch.2.012057 for calculation details, which includes Refs. [50,51].

[50] T. B. Liverpool, Phys. Rev. E 72, 021805 (2005).

[51] A. D. Polyanin and A. V. Manzhirov, Handbook of Integral Equations (CRC Press, Boca Raton, FL, 2008).

[52] G. Wilemski and M. Fixman, J. Chem. Phys. 60, 866 (1974).

[53] A notable exception to this Arrhenius law is the situation considered in Ref. [54] where the correlation time is infinite, contrarily to the present situation.

[54] I. Goychuk and P. Hänggi, Phys. Rev. Lett. 99, 200601 (2007).

[55] J. Pickands, Trans. Am. Math. Soc. 145, 75 (1969).

[56] M. Delorme, A. Rosso, and K. J. Wiese, J. Phys. A: Math Theor. 50, 16LT04 (2017).

[57] T. R. Powers, Rev. Mod. Phys. 82, 1607 (2010).

[58] O. Hallatschek, E. Frey, and K. Kroy, Phys. Rev. E 75, 031905 (2007).

[59] J. Shimada and H. Yamakawa, Macromolecules 17, 689 (1984).

[60] N. Douarche and S. Cocco, Phys. Rev. E 72, 061902 (2005).

[61] N. Becker, A. Rosa, and R. Everaers, Eur. Phys. J. E 32, 53 (2010).

[62] T. Guérin, Phys. Rev. E 96, 022501 (2017).

[63] S. Mehraeen, B. Sudhanshu, E. F. Koslover, and A. J. Spakowitz, Phys. Rev. E 77, 061803 (2008).

[64] R. Everaers, F. Jülicher, A. Ajdari, and A.C. Maggs, Phys. Rev. Lett. 82, 3717 (1999). 